\title{
Low-energy enhancement of nuclear $\gamma$ strength and its impact on astrophysical reaction rates
}

\author{
A. C. Larsen ${ }^{1, a}$, N. Blasi ${ }^{2}$, A. Bracco ${ }^{2,3}$, A. Bürger ${ }^{1}$, F. Camera ${ }^{2,3}$, T. K. Eriksen ${ }^{1}$, F. Giacoppo ${ }^{1}$, \\ S. Goriely ${ }^{4}$, M. Guttormsen ${ }^{1}$, A. Görgen ${ }^{1}$, T. W. Hagen ${ }^{1}$, S. Harissopulos ${ }^{5}$, P. E. Koehler ${ }^{1}$, S. \\ Leoni $^{2,3}$, B. Million ${ }^{2}$, H. T. Nyhus ${ }^{1}$, T. Renstrøm ${ }^{1}$, S. Rose ${ }^{1}$, I. E. Ruud ${ }^{1}$, A. Schiller ${ }^{6}$, S. Siem ${ }^{1}$, T. \\ Tornyi $^{1,7}$, G. M. Tveten ${ }^{1}$, A. V. Voinov ${ }^{8}$, and M. Wiedeking ${ }^{9}$
}

${ }^{1}$ Department of Physics, University of Oslo, N-0316 Oslo, Norway

${ }^{2}$ INFN, Sezione di Milano, Milano, Italy

${ }^{3}$ Dipartimento di Fisica, University of Milano, Milano, Italy

${ }^{4}$ Institut d'Astronomie et d'Astrophysique, ULB, Belgium

${ }^{5}$ Institute of Nuclear Physics, NCSR "Demokritos", Athens, Greece

${ }^{6}$ Norwegian Defence Research Establishment, N-2007 Kjeller, Norway

${ }^{7}$ Institute of Nuclear Research, MTA ATOMKI, H-4026 Debrecen, Hungary

${ }^{8}$ Department of Physics and Astronomy, Ohio University, Athens, Ohio 45701,USA

${ }_{9}^{9}$ Themba LABS, P.O. Box 722, 7129 Somerset West, South Africa

\begin{abstract}
An unexpected enhancement in the low-energy part of the $\gamma$-strength function for light and medium-mass nuclei has been discovered at the Oslo Cyclotron Laboratory. This enhancement could lead to an increase in the neutron-capture rates up to two orders of magnitude for very exotic, neutron-rich nuclei. However, it is still an open question whether this structure persists when approaching the neutron drip line.
\end{abstract}

\section{Introduction}

The $\gamma$-ray strength function characterizes average electromagnetic decay properties of excited nuclei. This quantity is indispensable for calculating nuclear reaction cross sections and reaction rates relevant for astrophysical applications. Recent studies [1,2] clearly show the importance of a precise description of the $\gamma$-ray strength function at low energies, especially for a proper understanding of the nucleosynthesis of the elements heavier than iron by the rapid neutron-capture process (r-process).

So far, however, the astrophysical site hosting such an r-process remains unknown, and therefore the physical conditions such as temperature and neutron-density profiles are not well determined. Apart from that, there is a wealth of nuclear data required, mostly involving very neutron-rich nuclei with extremely short life times. In the absence of an $(n, \gamma)-(\gamma, n)$ equilibrium, i.e. if the waitingpoint approximation is not fulfilled, the $(n, \gamma)$ reaction rates influence the element distribution as demonstrated in Ref. [2]. As the $(n, \gamma)$ reaction rate strongly depends on the $\gamma$-ray strength function, a good understanding of this quantity is vital.

\footnotetext{
ae-mail: a.c.larsen@fys.uio.no
} 
The nuclear physics group in Oslo has performed measurements on the $\gamma$-ray strength functions below neutron threshold of various light and medium-mass nuclei (see, e.g., [3-5]). These data have revealed an unexpected increase in the $\gamma$-decay probability at low $\gamma$-ray energies. This enhancement is seen to be present typically for $E_{\gamma} \leq 3 \mathrm{MeV}$. In contrast to other soft resonances observed previously such as the $M 1$ scissors mode [6], the physical origin of the enhancement remains unknown. There is, for the time being, no established theory that is able to explain this phenomenon (see Fig. 1, left), and it is not known whether this enhancement is present in neutron-rich nuclei.
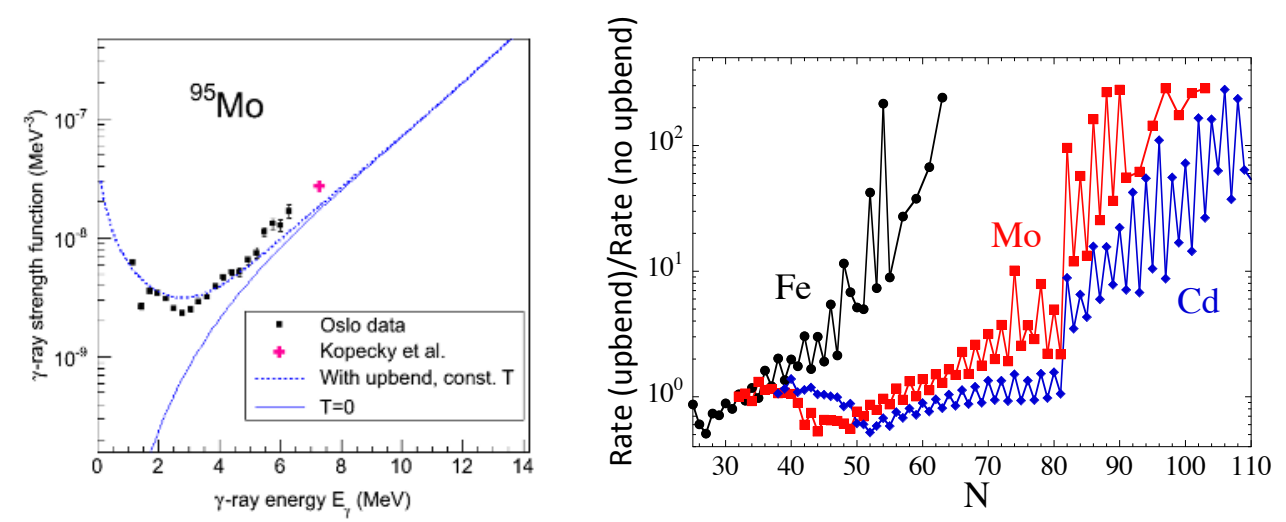

Figure 1. Left: Oslo data of the $\gamma$-strength function of ${ }^{95} \mathrm{Mo}$ (black squares, from Ref. [4]), and models of the strength assuming zero temperature (solid, blue line) and with a constant temperature $T_{f}=0.30 \mathrm{MeV}$, including also a parameterization of the upbend (dashed line). Right: Ratios of $(n, \gamma)$ reaction rates at $T=1 \cdot 10^{9} \mathrm{~K}$, with and without the upbend in the $\gamma$ strength, for $\mathrm{Fe}, \mathrm{Mo}$, and $\mathrm{Cd}$ isotopes approaching the neutron drip line [7].

\section{Experimental details}

The experiments were performed at the Oslo Cyclotron Laboratory (OCL) using a light-ion beam delivered by the MC-35 Scanditronix cyclotron. Self-supporting targets enriched to $\approx 95 \%$ in the isotope of interest, and with a thickness of $\approx 2 \mathrm{mg} / \mathrm{cm}^{2}$ were placed in the center of the multi-detector array CACTUS [8]. CACTUS consists of 28 collimated $5^{\prime \prime}$ by $5^{\prime \prime} \mathrm{NaI}(\mathrm{Tl}) \gamma$-ray detectors with a total efficiency of $15.2(1) \%$ for $E_{\gamma}=1332 \mathrm{keV}$. For the experimental campaign on the iron isotopes ${ }^{56,57} \mathrm{Fe}$ carried out in March 2012, six of the NaI crystals were replaced with large-volume (3.5" by $\left.8^{\prime \prime}\right) \mathrm{LaBr}_{3}(\mathrm{Ce})$ detectors from the INFN Milan group. The CACTUS setup is shown in Fig. 2 (left).

The charged ejectiles were measured with the Silicon Ring (SiRi) particle-detector system [9]. The SiRi system consists of eight $130-\mu \mathrm{m}$ thick silicon detectors, each of which is divided into eight strips. One strip has an angular resolution of $\Delta \theta=2^{\circ}$. Each of these segmented, thin detectors are put in front of a $1550-\mu \mathrm{m}$ thick back detector. The full SiRi system covers scattering angles between $40-54^{\circ}$ when placed in forward direction and a solid-angle coverage of $\approx 6 \%$.

The particle- $\gamma$ coincidences were sorted into excitation-energy versus $\gamma$-ray energy matrices, as shown for ${ }^{57} \mathrm{Fe}$ in the right panel of Fig. 2 . Then the $\gamma$ spectra were unfolded with the known response functions of CACTUS as described in Ref. [10]; newly measured response functions were used for the $\mathrm{LaBr}_{3}$ detectors. From the unfolded $\gamma$ spectra, the distribution of primary $\gamma$ rays were extracted from the full cascades by an iterative subtraction technique [11]. Further, the level density and $\gamma$ strength 

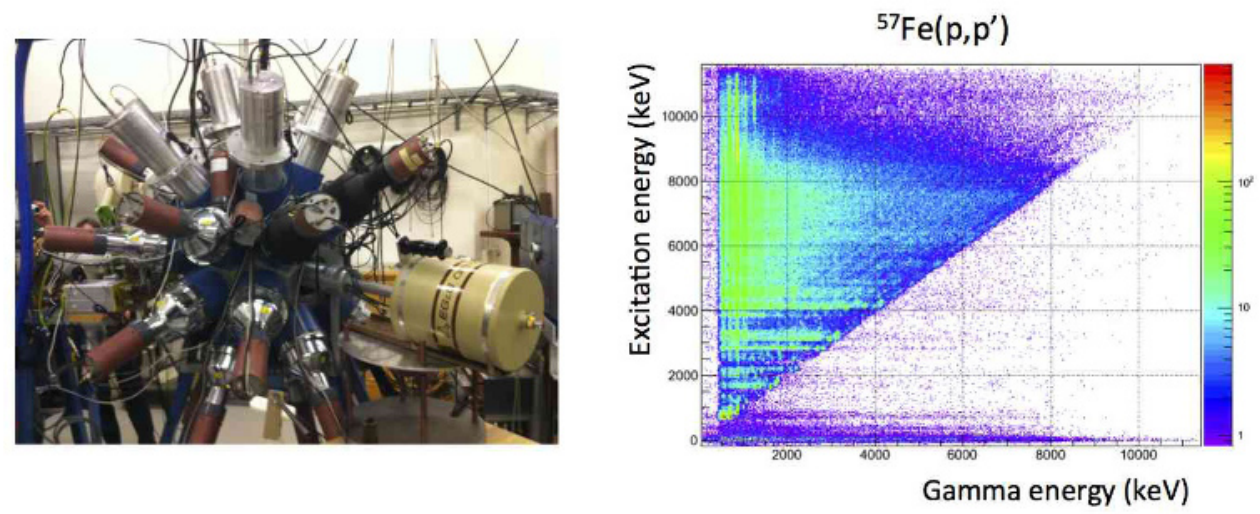

Figure 2. Left: The $\gamma$-detector array CACTUS, including six large-volume $\mathrm{LaBr}_{3}(\mathrm{Ce})$ scintillators from the INFN Milan group. Right: Proton- $\gamma$ coincidence matrix from the ${ }^{57} \mathrm{Fe}\left(\mathrm{p}, \mathrm{p}^{\prime}\right)$ experiment at OCL, March 2012 (beam energy $E_{p}=16 \mathrm{MeV}$ ).

were determined from a simultaneous fit of the primary $\gamma$ matrix [12]. For more details, see also Ref. [13].

\section{Discussion of results}

As shown in Ref. [7] and in the right panel of Fig. 1, the low-energy enhancement in the $\gamma$ strength has the potential to boost the Maxwellian-averaged neutron-capture cross sections up to two orders of magnitude. Very recently the existence of the upbend pattern has been independently confirmed in ${ }^{95} \mathrm{Mo}$, see Ref. [14], but there is still no experimental data of the $\gamma$-strength function in neutron-rich nuclei. It would be of utmost importance to obtain such data in order to improve on the neutroncapture rates in this region.

There is a great need for a better understanding of the enhancement itself. At present, the multipolarity, the electromagnetic character, and the mechanism behind this structure is unknown. However, data on ${ }^{60} \mathrm{Ni}$ might indicate an $M 1$ nature [15]. With new data on ${ }^{56,57} \mathrm{Fe}$, using also six $3.5^{\prime \prime}$ by $8^{\prime \prime}$ $\mathrm{LaBr}_{3}(\mathrm{Ce})$ scintillators borrowed from the INFN Milan group, one expects to be able to determine the multipolarity of the low-energy enhancement in these nuclei from angular distributions. These data sets clearly confirm the presence of the low-energy enhancement as shown for ${ }^{56} \mathrm{Fe}$ in Fig. 3, using another beam species, beam energy, and with a greatly improved excitation-energy and $\gamma$-energy resolution compared to the previous experiments in Ref. [3].

Also, it is not clear in which mass region the enhancement is present. New data on Cd isotopes indicate that the lighter isotopes ${ }^{105,106} \mathrm{Cd}$ appear to have more $\gamma$ strength for $E_{\gamma}<3 \mathrm{MeV}$ than the heavier ${ }^{111,112} \mathrm{Cd}$. It could be that this is the transitional region for the low-energy enhancement, which has been seen for ${ }^{93-98}$ Mo but not for ${ }^{116} \mathrm{Sn}$ and heavier nuclei. However, more data on nuclei in this intermediate mass region is needed before any firm conclusions can be drawn. The analysis of several $\mathrm{Pd}$ isotopes is currently ongoing. 


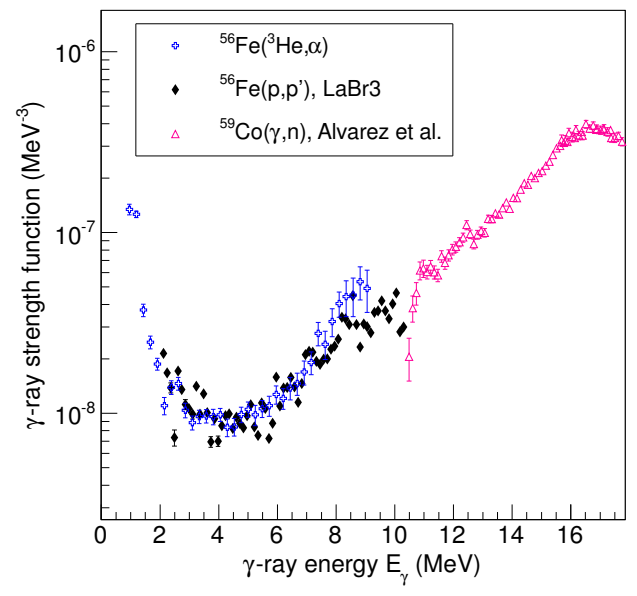

Figure 3. The $\gamma$-ray strength function of ${ }^{56} \mathrm{Fe}$ from the recent $\left(p, p^{\prime}\right)$ experiment using $\operatorname{LaBr}_{3}(\mathrm{Ce})$ detectors for the $\gamma$ rays (black diamonds), from the $\left({ }^{3} \mathrm{He}, \alpha\right)$ data [3] (open, blue crosses), together with ${ }^{59} \operatorname{Co}(\gamma, n)$ data from Ref. [16] as there are no experimental ${ }^{56} \mathrm{Fe}(\gamma, n)$ data available.

\section{Summary and outlook}

The discovery of an enhancement in the $\gamma$-strength function at low $\gamma$ energies using the Oslo method has drawn much attention recently. It has been confirmed by an independent method, it has turned out to be potentially crucial for astrophysical neutron-capture rates, and it has initiated much theoretical activity. New data on Cd isotopes indicate that the transitional region could be around $A \sim 105$, but more data is needed to confirm this. We have proposed an experimental campaign at HIE-ISOLDE to measure the $\gamma$-strength function in neutron-rich medium-mass nuclei, starting with a ${ }^{66} \mathrm{Ni}$ beam as a benchmark test, which has recently been approved. It is anticipated that this experimental campaign will reveal whether the low-energy enhancement is present for more neutron-rich nuclei.

\section{References}

[1] M. Arnould, S. Goriely, and K. Takahashi, Phys. Rep. 450, 97 (2007).

[2] S. Goriely, Phys. Lett. B436, 10 (1998).

[3] A. Voinov et al., Phys. Rev. Lett 93, 142504 (2004).

[4] M. Guttormsen et al., Phys. Rev. C 71, 044307 (2005).

[5] A. C. Larsen et al., Phys. Rev. C 76, 044303 (2007).

[6] A. Schiller et al., Phys. Lett. B 633, 225 (2006).

[7] A. C. Larsen and S. Goriely, Phys. Rev. C 82, 014318 (2010).

[8] M. Guttormsen et al., Phys. Scr. T 32, 54 (1990).

[9] M. Guttormsen et al., Nucl. Instrum. Methods Phys. Res. A 648, 168 (2011).

[10] M. Guttormsen et al., Nucl. Instrum. Methods Phys. Res. A 374, 371 (1996).

[11] M. Guttormsen et al., Nucl. Instrum. Methods Phys. Res. A 255, 518 (1987).

[12] A. Schiller et al., Nucl. Instrum. Methods Phys. Res. A 447498 (2000).

[13] A. C. Larsen et al., Phys. Rev. C 83, 034315 (2011).

[14] M. Wiedeking et al., Phys. Rev. Lett. 108, 162503 (2012).

[15] A. Voinov et al., Phys. Rev. C 81, 024319 (2010).

[16] R. A. Alvarez et al., Phys. Rev. C 20, 128 (1979). 